\title{
Verifying Relationship between fovea Palatini, Vibrating Lines and Junction between Hard and Soft Palate
}

\author{
Dr Dipak Thapa,' Dr Suresh Chandra,, Dr Sunita Karki \\ 'Faculty, ${ }^{2}$ Professor, ${ }^{3} \mathrm{PG}$ Resident, Dept of Prosthodontics, Kantipur Dental College, Kathmandu, Nepal
}

Correspondence: Dr Dipak Thapa; Email: drdthapa@hotmail.com

\section{ABSTRACT}

Introduction: Locating and incorporating posterior palatal seal (PPS) in maxillary denture is an important clinical procedure for retention. The use of anterior and posterior vibrating lines, hard and soft palate junction, fovea palatini and hamular notches as references to locate PPS, are largely disputed.

Objective: To verify the relationship between fovea palatini, anterior and posterior vibrating line, and hard and soft palate junction and their role in locating PPS area.

Materials \& Method: 50 subjects were randomly selected. In each subject, hard and soft palate junction, anterior and posterior vibrating lines and fovea palatini were located and marked. Using Vernier caliper and divider, the distance between these lines and fovea palatini were measured and assessed.

Result: $\vee 44 \%$ showed fovea palatini at hard and soft palate junction, $24 \%$ between junction and anterior vibrating line/vibrating line, $6 \%$ at anterior vibrating line, $24 \%$ between anterior and posterior vibrating lines and $2 \%$ at posterior vibrating line. In $44 \%$, hard and soft palate junction coincided with anterior vibrating line. In the rest, mean distance was $2.76 \mathrm{~mm}$ right, $2.38 \mathrm{~mm}$ mid line and $2.79 \mathrm{~mm}$ left. $20 \%$ subjects revealed one vibrating line; $80 \%$ subjects had two vibrating lines with the mean distance 5.55 $\mathrm{mm}$ on right, $6.22 \mathrm{~mm}$ mid line and $5.40 \mathrm{~mm}$ on left.

Conclusion: Recording posterior palatal seal is an important clinical step thus arbitrarily scraping of the cast using fovea palatini and hamular notch as reference, should be discouraged. Locating anterior and posterior vibrating lines and using them to determine the posterior extension and PPS should be recommended.

Keywords: anterior vibrating line, fovea palatini, hard and soft palate junction, posterior vibrating line

\section{INTRODUCTION}

The location and achievement of Posterior Palatal Seal (PPS) though important has so far remained a highly controversial issue as the searching of literature reveals various methods; viz: 1. the arbitrary technique of scraping the cast before processing, 2. selective loading impression technique and 3. physiological impression technique.' Even in these methods there are great variations regarding the pattern (single bead/ double bead/ butterfly), depth of scraping (0.5 -1.5 mm), material use (wax/green stick) and stage of recording (during border moulding/after final impression/ during trial denture/ during denture delivery).

One of the easiest and most commonly practiced method for determining the extent of posterior palatal seal and that of the maxillary complete denture is using the anatomical landmarks (fovea palatini and hamular notch). ${ }^{2,3}$ However there is great variation regarding the position of the fovea palatine as $1.31 \mathrm{~mm}$ anterior to the anterior vibrating line (Lye), ${ }^{4}$ either on or behind the anterior vibrating line (Chen), ${ }^{5}$ about $2 \mathrm{~mm}$ behind the vibrating line, which is posterior vibrating line as it is produced during phonation of "ah" sound (Zarb Bolender). ${ }^{6}$ Chen thus concluded that the fovea palatini are not reliable guides for locating posterior border of the maxillary denture. ${ }^{5}$

The accepted number of vibrating lines also varies. According to Rashedi et al;' 95\% American dental schools believe 'one vibrating line concept' (i.e. posterior vibrating line) where the posterior termination of maxillary denture is done on this vibrating line. Zarb Bolender ${ }^{6}$ too advocated one vibrating line concept; he recommended that the distal end of the denture should be extended at least to the vibrating line and in most instances, it should end 1-2 $\mathrm{mm}$ posterior to the vibrating line (Figure 1). Furthermore, Silverman ${ }^{7}$ stated that the PPS could be extended further back (dorsally) than the 
Figure 1: Anatomical landmarks of maxilla

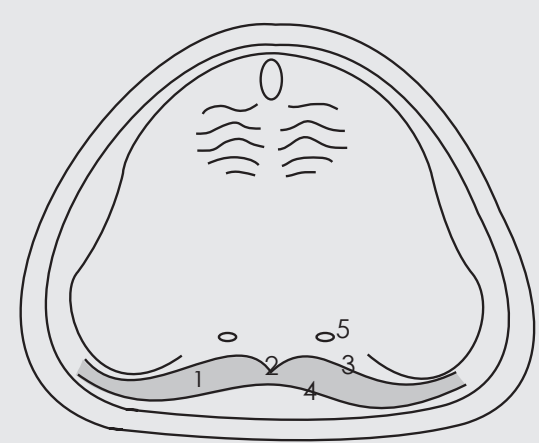

1 Junction between hard palate and soft palate 2 Posterior nasal spine

3 Anterior vibrating line

4 Posterior vibrating line

5 Fovea palatini vibrating line by about $8.2 \mathrm{~mm}$ to substantially aid in retention and stability of the upper denture. However certain schools in US and locally most of the dental schools in Nepal and India follow two vibrating line concept, and PPS is recorded between these two vibrating lines.

Rahn ${ }^{8}$ stated that hard and soft palate junction can be best determined by having the patient close both nostrils and blow gently (Valsalva maneuver). However Winkler ${ }^{9}$ recommended this Valsalva maneuver for locating anterior vibrating line and this procedure will position soft palate inferiorly at its junction with hard palate, and due to projection of the posterior nasal spine, this line is not a straight line between both hamular processes. He further stressed that this anterior vibrating line should not be confused with the anatomic junction of hard and soft palate.

Therefore, to verify the location of fovea palatini, to find the existence of one or more vibrating lines and their relationship with the junction between the hard and soft palate, together their role in PPS, a clinical study was conducted on both dentulous and edentulous subjects.

\section{MATERIALS AND METHOD}

This study was conducted in the Department of Prosthodontics, Kantipur Dental College \& Hospital during SeptemberDecember 2015. The study was conducted after receiving ethical clearance from Institutional Review Board. The study was cross sectional, non-interventional and observational with randomly selected fifty subjects; out of which 10 were edentulous. Each subject was asked to seat comfortably on a dental chair in upright position. At first, hard and soft palate junction was palpated with the help of "T" burnisher and marked with indelible pencil. Then Valsalva maneuver was preformed to locate and mark anterior vibrating line where the subject was asked to close both the nostrils and blow gently. The phonation of "ah" sound was used to locate posterior vibrating line which was also marked with indelible pencil. At the end, fovea palatini was identified, one on each side of the mid line, then marked and connected with a straight line.

The location of the fovea with respect to junction and vibrating lines was noted and measured at mid line using scale and the divider. Distance between the junction and anterior vibrating line and distance between the anterior and posterior vibrating lines were also measured at three points; one at mid line, another at the right side (midway between right hamular and midline), and lastly at the left side (midway between left hamular and midline).

\section{RESULT}

In the present study, 23 subjects out of $50(46 \%)$ had fovea palatini on junction between hard and soft palate. Out of these 23 subjects, 12 subjects had their anterior vibrating line also coincided with the junction. $10(20 \%)$ subjects had fovea between the junction and the anterior vibrating line/vibrating line. The mean distance of fovea from the junction was $2.72 \mathrm{~mm}$ posterior or on other way fovea was $2.44 \mathrm{~mm}$ anterior to the anterior vibrating line/vibrating line. $3(6 \%)$ subjects had fovea on the anterior vibrating line. $12(24 \%)$ subjects had fovea between anterior and posterior vibrating line. In $1(2 \%)$ subject fovea coincided with posterior vibrating line while the anterior vibrating line coincided with the junction (Table 1).

Table 1: Location and distance of fovea palatini

\begin{tabular}{|l|c|c|c|c|c|c|}
\hline \multicolumn{1}{|c|}{ Score } & Anterior to the junction & On the junction & $\begin{array}{c}\text { Between junction } \\
\text { and AVL/VL (mm) }\end{array}$ & On AVL & $\begin{array}{c}\text { Between AVL } \\
\text { and PVL (mm) }\end{array}$ & \begin{tabular}{c} 
On PVL \\
\hline No of subjects
\end{tabular} \\
\hline Mean distance & 0 & $23(46 \%)$ & $10(20 \%)$ & $2(4 \%)$ & $14(28 \%)$ & $1(2 \%)$ \\
\hline
\end{tabular}

Table 2: Distance between different anatomical lines

\begin{tabular}{|l|c|c|c|c|c|c|c|}
\hline \multirow{2}{*}{} & \multicolumn{3}{|c|}{ Distance between Junction and AVL $(\mathbf{m m})$} & \multicolumn{3}{c|}{ Distance between AVL and PVL (mm) } \\
\cline { 2 - 8 } & Right & Mid & Left & Right & Mid & Left \\
\hline Mean distance & 2.76 & 2.38 & 2.79 & 5.55 & 6.22 & 5.40 \\
\hline
\end{tabular}


In $10(20 \%)$ subjects there was only one vibrating line. In $80 \%$ subjects there were 2 vibrating lines. In 23 subjects, the junction between the hard and soft palate and the anterior vibrating line coincided. In the rest the mean distance between junction and hard palate was: at right side $2.76 \mathrm{~mm}$, in mid line $2.38 \mathrm{~mm}$ and in left side $2.79 \mathrm{~mm}$. The mean distance between the anterior vibrating line and posterior vibrating lines were: right side $5.55 \mathrm{~mm}$, midline $6.22 \mathrm{~mm}$ and on left side $5.40 \mathrm{~mm}$ (Table 2).

\section{DISCUSSION}

It is true that there is a great variation regarding the position of fovea palatini. Most of the subjects in our study had fovea on the junction between hard and soft palate. However, Chen found that majority of the subjects had fovea palatini on or behind the anterior vibrating line. ${ }^{5}$ In the present study; only $4 \%$ had fovea palatini on the anterior vibrating line and $30 \%$ behind the anterior vibrating line.

Majority of the subjects i.e. $66 \%$ in our study had fovea anterior to the anterior vibrating line. According to Lye, fovea palatini is located $1.31 \mathrm{~mm}$ anterior to anterior vibrating line. ${ }^{4}$ In our study it is located $2.44 \mathrm{~mm}$ anterior to the anterior vibrating line (but within the soft palate). According to Lye; anterior vibrating line is an imaginary line that marks the beginning of motion of the soft palate. According to Winkler; anterior vibrating line is an imaginary line located at the junction of the attached tissue overlying the hard palate and the movable tissue of immediately adjacent soft palate..$^{9}$ If these statements are to be considered; then fovea palatini lie within the soft tissue covering the hard palate. But in our study no single subject had the fovea anterior to the junction.
According to Bolender, fovea lies about $2 \mathrm{~mm}$ behind the vibrating line (which is posterior vibrating line as it is produced during the phonation of 'ah' sound). ${ }^{6}$ We found only one subject whose fovea was on the posterior vibrating line but there was none in whom fovea was present behind the posterior vibrating line.

According to Rashedi et al' most of the dental school in US are teaching 'one vibrating line' concept but $80 \%$ of our subjects had two vibrating lines. Out of these $80 \%, 23$ subjects had junction and anterior vibrating line coincided.

\section{CONCLUSION}

Recording the posterior palatal seal is an important clinical step during fabrication of maxillary complete denture. Therefore arbitrarily scraping of the cast by lab technician and depending on fovea palatini and hamular notch for locating posterior palatal seal (PPS) area should be discouraged. Locating anterior and posterior vibrating lines and using them to determine the posterior extension and PPS should be recommended.

\section{Acknowledgement:}

Authors would like to thank interns Dr Ram Kumar Adhikari, Dr Sumita Suwal, Dr Chetan Basnet and Dr Sneha Adhikari for their contribution in data collection.

\section{REFERENCES}

1. Rashedi B, Petropoulos V. Current concepts for determining the postpalatal Seal in Complete Dentures. J Prosthodont. 2003; 12:265-70.

2. Yasmen A. Reliability of fovea palatini in determining the posterior palatal seal. J Bagh College Dentistry. 2009; 21 (1):41-5.

3. Bhayana R, Jain SR. Fovea Palatini- what lies beneath and down under. J Dentofacial Sciences. 2013;2(3):47-50.

4. Lye TL. The significance of the fovea palatini in complete denture prosthodontics. J Prosthet Dent. 1975; 33:504-10.

5. Chen MS, Welker WA. Methods taught in dental schools for determining the posterior palatal seal region. J Prosthet Dent. 1985; 53:380-3.

6. David M Davis: Prosthodontic Treatment for Edentulous Patients. Zarb Bolender. 2005, pp 217.

7. Silverman SI: Dimension and displacement patterns of the posterior palatal seal. J Prosthet Dent. 1971; 25:470-88.

8. Rahn AO, Heartwell CM Jr: Textbook of Complete Dentures (ed5). Lea \& Febiger, Philadelphia. $1993, \mathrm{pp} 13$.

9. Winkler S: Essentials of Complete Denture Prosthodontics (ed2). Ishiyaku EuroAmerica Inc, 2000, pp 111. 